\author{
P. M. Spieth \\ H. Zhang
}

\section{Analyzing lung crackle sounds: stethoscopes and beyond}

Received: 2 June 2011

Accepted: 2 June 2011

Published online: 29 June 2011

(C) Copyright jointly held by Springer and ESICM 2011

This editorial refers to the article available at: doi:10.1007/s00134-011-2291-4.

\section{P. M. Spieth (}

Department of Anesthesia and Intensive Care Therapy, University Hospital Dresden, 01307 Dresden, Germany

e-mail: peter.spieth@uniklinikum-dresden.de

Tel.: +49-351-4582785

Fax: +49-351-4584336

\section{H. Zhang}

The Keenan Research Centre, Li Ka Shing Knowledge Institute, St. Michael's Hospital, Toronto, ON, Canada

Assessing the pulmonary function by physical examination is as old as the field of respiratory medicine. In 1819 the French physician René-Théophile-Hyacinthe Laennec (1781-1826) published his milestone book De l'Auscultation Médiate ou Traité du Diagnostic des Maladies des Poumons et du Coeur introducing the stethoscope as a diagnostic tool for the bedside assessment of respiration [1]. Even today, the stethoscope represents a symbol for physicians of all specialties. Despite all technical advances in recent years, auscultation provides both useful physiological information and close patient-physician interaction. Although auscultation is considered to be a very useful bedside tool, its major limitations are high interobserver variability, difficult description and documentation of findings, and the high dependency on individual expertise in acquiring and interpreting auscultation findings. Obtaining and interpreting lung sounds and especially adventitious sounds are a true art [2]. Secondary to its role in routine physical examination, the analysis of lung sounds can be used in the intensive care setting to gather information about the performance and efficiency of mechanical ventilation prior to or instead of more invasive radiological procedures like chest X-rays or computer tomography (CT).

In 1994 Suki et al. [3] described an avalanche-like phenomenon of discrete jumps in airway resistance following a power law distribution of airway opening during constant flow inflation in isolated dog lungs. They proposed that the recruitment of terminal airspaces is governed by this avalanche phenomenon associated with threshold phenomena propagating down a branching tree structure [3]. Several years later the same group modeled the inflation of collapsed regions during inspiration using the concept of the avalanches propagating through a bifurcating network of airways [4]. They described the avalanche "shocks" as a cascade of dynamic pressure instabilities manifested as negative elastic resistance of the lung [4]. The complex phenomenon of avalanche-like recruitment of distal airways could also be validated by using lung crackle sounds [5].

Today there are automated systems available to detect, analyze, and interpret lung sounds [6]. Image-based techniques of lung sound analysis such as vibration response imaging have been introduced and tested clinically, e.g., in patients with pneumonia [7] and to predict postoperative lung function following lung resection [8]. However, these techniques are still highly experimental and not routinely used. Experimental and clinical studies are warranted to determine their potential role in clinical practice.

In this issue of Intensive Care Medicine, Dr. Vena et al. [9] report a nicely performed analysis of the spectral characteristics of lung sounds. The objective of the study was to develop a simple tool to use lung sound analysis for detection of lung injury and alveolar recruitment. 
Standard CT assessment of lung aeration was performed to validate these findings. In addition to the spectral analysis and the CT exams they further confirmed their findings by conventional interpretation of the lung sounds by experienced physicians. It is worth noting that the significant correlation between intratidal recruitment measured with dynamic CT and the degree of crackles in the sound analysis suggests that air passage through atelectasis as well as poorly aerated areas is crucial for generating lung crackle sounds [9]. Therefore the authors proved that crackles are generated and not limited in fully collapsed lung areas. Dr. Vena et al. concluded that the computer-based analysis of crackle sounds is more sensitive to differentiate between small differences in healthy and injured conditions especially at higher positive end- expiratory pressure (PEEP) levels compared to conventional clinical auscultation.

This simple and noninvasive technique of computerbased lung sound analysis may help intensivists monitor mechanical ventilation and diagnostic or therapeutic procedures such as recruitment maneuvers in real time at the bedside. Yet the validation of the technique, and the variation of frequent measurements and interpretation of complex respiratory signals to optimize ventilator settings or to detect alveolar recruitment, especially in injured lungs, remain to be further investigated at the bedside [10]. The automated analysis of lung sounds may be used in the near future to translate Laennec's brilliant idea into modern intensive care.

\section{References}

1. Laennec R (1819) De l'Auscultation Médiate ou Traité du Diagnostic des Maladies des Poumons et du Coeur. Brosson \& Chaudé, Paris

2. Forgacs P (1967) Crackles and wheezes. Lancet 2:203-205

3. Suki B, Barabsi AL, Hantos Z, Petk F, Stanley HE (1994) Avalanches and power-law behavior in lung inflation. Nature 368:615-618

4. Alencar AM, Arold SP, Buldyrev SV, Majumdar A, Stamenovic D, Stanley HE, Suki B (2002) Physiology: dynamic instabilities in the inflating lung. Nature 417:809-811

5. Alencar AM, Buldyrev SV, Majumdar A, Stanley HE, Suki B (2001) Avalanche dynamics of crackle sound in the lung 1. Phys Rev Lett 87:088101
6. Abbas A, Fahim A (2010) An automated computerized auscultation and diagnostic system for pulmonary diseases. J Med Syst 34:1149-1155

7. Bartziokas K, Daenas C, Preau S, Zygoulis P, Triantaris A, Kerenidi T, Makris D, Gourgoulianis KI, Daniil Z (2010) Vibration response imaging: evaluation of rater agreement in healthy subjects and subjects with pneumonia. BMC Med Imaging 10:6

8. Morice RC, Jimenez CA, Eapen GA, Mehran RJ, Keus L, Ost D (2010) Using quantitative breath sound measurements to predict lung function following resection. J Cardiothorac Surg 5:81
9. Vena A, Rylander C, Perchiazzi, G, Giuliani R, Hedenstierna G (2011) Lung sound analysis correlates to injury and recruitment as identified by computed tomography: an experimental study. Intensive Care Med 37. doi: 10.1007/s00134-011-2291-4

10. Carvalho AR, Spieth PM, Pelosi P, Vidal Melo MF, Koch T, Jandre FC, Giannella-Neto A, de Abreu MG (2008) Ability of dynamic airway pressure curve profile and elastance for positive end-expiratory pressure titration. Intensive Care Med 34:2291-2299 